\title{
Rehabilitation following lumbar fusion surgery (REFS) a randomised controlled feasibility study
}

\author{
James Greenwood ${ }^{1}\left[\right.$ D $\cdot$ Alison McGregor ${ }^{2} \cdot$ Fiona Jones $^{3} \cdot$ Michael Hurley $^{3}$
}

Received: 1 November 2018 / Revised: 4 February 2019 / Accepted: 6 February 2019 / Published online: 20 February 2019

(c) The Author(s) 2019

\begin{abstract}
Purpose Following lumbar fusion surgery (LFS), $40 \%$ of patients are unsure/dissatisfied with their outcome. A prospective, single-centre, randomised, controlled trial was conducted to evaluate the feasibility (including clinical and economic impact) of a theoretically informed rehabilitation programme following LFS (REFS).

Methods REFS was informed by an explicit theoretical framework and consisted of 10 consecutive weekly group rehabilitation sessions (education, low-tech cardiovascular, limb and spine strengthening exercises, and peer support). Participants were randomised to REFS or 'usual care.' Primary feasibility outcomes included recruitment and engagement. Secondary outcomes, collected preoperatively and 3, 6, and 12 months postoperatively, comprised the Oswestry disability index, European Quality of Life 5 dimensions score, pain self-efficacy questionnaire, hospital anxiety and depression scale and the aggregated functional performance time. Economic impact was evaluated with the Client Services Receipt Inventory.

Results Fifty-two of 58 eligible participants were recruited, and engagement with REFS was $>95 \%$. REFS participants achieved a clinically meaningful reduction in unadjusted mean short-term disability $(-13.27 \pm 13.46)$, which was not observed in the 'usual care' group $(-2.42 \pm 12.33)$. This was maintained in the longer term $(-14.72 \% \pm 13.34 \mathrm{vs}-7.57 \pm 13.91)$. Multilevel regression analyses, adjusted for body mass index, baseline depression, and smoking status reported a statistically significant short-term improvement in disability $(p=0.014)$ and pain self-efficacy $(p=0.007)$. REFS costs $£ 275$ per participant.
\end{abstract}

Conclusions Results suggest that REFS is feasible and potentially affordable for delivery in the National Health Service. It is associated with a clinically meaningful impact. A multicentre randomised controlled study to further elucidate these results is warranted.

\section{Graphical abstract}

These slides can be retrieved under Electronic Supplementary Material.
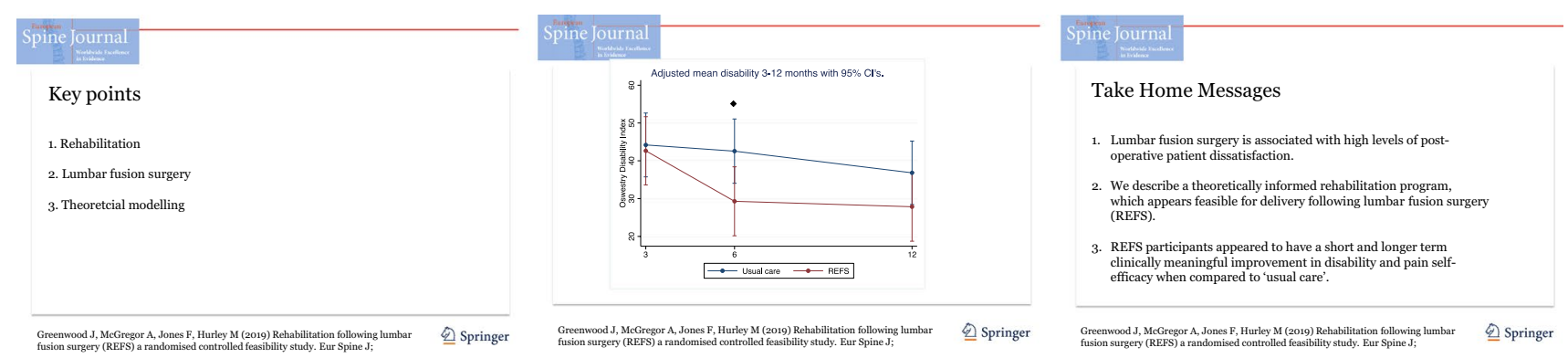

Keywords Lumbar fusion surgery $\cdot$ Rehabilitation $\cdot$ Theoretical modelling $\cdot$ Physiotherapy

Electronic supplementary material The online version of this article (https://doi.org/10.1007/s00586-019-05913-6) contains supplementary material, which is available to authorized users.

Extended author information available on the last page of the article 


\section{Introduction}

Lumbar fusion surgery (LFS) is commonly undertaken in parallel with the decompression of affected neural tissue to relieve back/leg symptoms. Common indications include spondylolisthesis, disc disease, and stenosis [1]. The volume of LFS is increasing particularly in patients over 60 years [2]. In high income countries, the population of those over 60 is growing faster than any other age group [3]. Therefore, the increase in LFS is likely to continue, with patients living longer postoperatively.

Forty per cent of all patients are unsure/dissatisfied with their outcome following LFS [4], experiencing impaired psychological, social, and neuromusculoskeletal function [5]. Many of these reported problems appear amenable to rehabilitation. A recent meta-analysis demonstrated that 'complex' rehabilitation (exercise combined with psychologically mediated content, such as cognitive behavioural therapy) enhanced outcome when compared with 'usual care' [6]. However, the included studies [7], and [8], had methodological constraints limiting the wider extrapolation of these results. Both studies commenced rehabilitation within 3 weeks of LFS, potentially contributing to the high $(22.6 \%)$ re-operation rate [7]. Other researchers have demonstrated inferior outcomes with rehabilitation commencing before 3 months [9]. Abbott et al. [7] excluded participants over 65 years of age, and the 'usual care' comparator group consisted of a single session (20 $\mathrm{min})$ of exercise-based self-management advice. This does not reflect current 'usual care' in the UK, which typically consists of 6 sessions of individualised physiotherapy [10]. In the study by [8], the 'psychomotor' rehabilitation intervention comprised $38 \mathrm{~h}$ of hospital-based, multi-professional rehabilitation (physiatrists, psychologists, and physiotherapists). Whilst no economic reports were identified, it is plausible such a programme would prove prohibitively costly to deliver. Therefore, previous studies [7, 8] are not representative of those patients undergoing LFS in the UK.

Guidance from the Medical Research Council (MRC) encourages the use of theoretical modelling in the early development of complex healthcare interventions, such as rehabilitation [11]. Despite this, neither study from the meta-analysis was based on any explicit theoretical framework. Currently, no evidence-based 'gold standard' rehabilitation regime exists following LFS. To our knowledge, no study has compared a theoretically informed rehabilitation programme with current UK 'usual care' (individual physiotherapy). To bridge this research gap, there is an urgent need to develop and evaluate a rehabilitation programme that is acceptable to participants, safe, and affordable for use following LFS [6].
The design and evaluation of the rehabilitation programme in this study, rehabilitation following lumbar fusion surgery (REFS), was guided by the checklist for group-based behavioural change interventions [12] and the Consolidated Standards of Reporting Trials and extension for non-pharmacological trials (CONSORT) [13].

The primary aim of the study was to evaluate the feasibility of delivering the REFS programme following technically successful LFS. The secondary aim was to describe the clinical and economic impact of REFS compared with 'usual care.'

\section{Materials and methods}

\section{Study design}

This was a prospective, single-centre, randomised controlled feasibility study. In keeping with MRC guidance [11], a theoretical framework was developed to inform the content and delivery aspects of REFS using the behavioural change wheel methodology [14] and social cognitive theory (SCT) [15]. The resulting hypothetical causal pathway (Fig. 1) and REFS programme are presented (Fig. 2). The study was approved by the National Research Ethics Service (NRES) at Queen Square on the 14th June 2014 (14/LO/0748) and registered, ISRCTN60891364.

\section{Participants}

A convenience sample $(n=52)$ of participants was recruited between August 2014 and April 2016. Potential participants were identified from the neurosurgical waiting list, screened for eligibility, sent a patient information sheet, and were met preoperatively to discuss participation. Those willing to enrol in the study provided signed consent and baseline data.

Participants were considered eligible if they were aged 18-75 years and undergoing primary LFS (1-3 inter-vertebral levels) for degenerative/congenital conditions. Participants were excluded if they had previously undergone LFS (prior laminectomy/discectomy were eligible); had spinal cord involvement; lower limb joint pain precluding assessment; could walk less than 20 metres on flat ground; had poorly controlled psychological/medical comorbidity limiting engagement with rehabilitation; inadequate verbal and written English language skills; or were unwilling/unable to undertake exercise, attend REFS or provide consent. Block randomisation was employed (Sealed Envelope Ltd, https:// www.sealedenvelope.com, accessed 22/6/2014) (REFS or 'usual care'). Codes were generated at the remote host University site and placed into sequentially numbered opaque envelopes to preserve blinding of allocation. The nature of the intervention prevents the blinding of participants, 


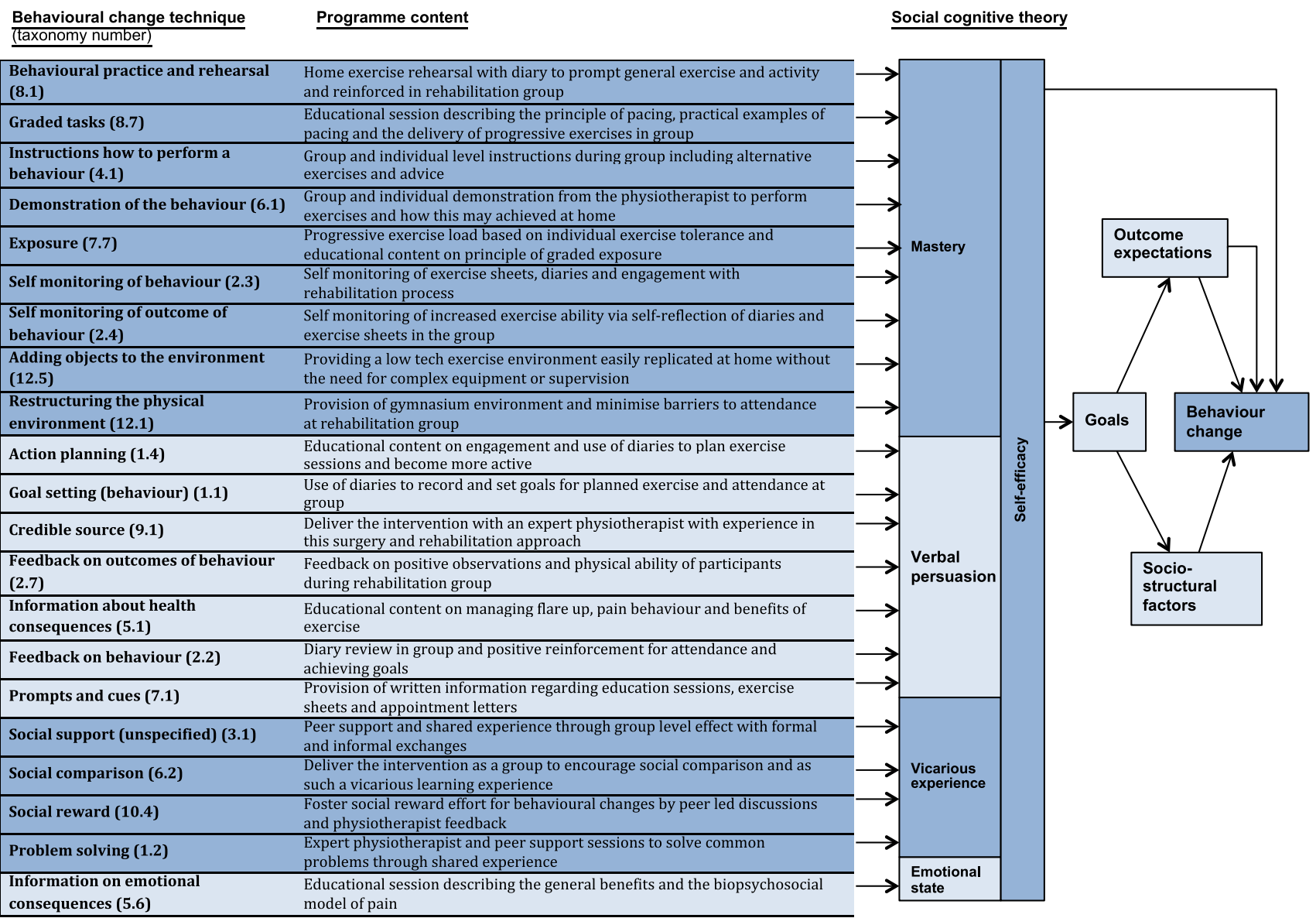

Fig. 1 Hypothetical causal pathway informing the development of the REFS programme

who were informed of their allocation. Data collection and study physiotherapists were not blinded to allocation. As the majority of outcome measures were self-reported, this potential source of bias was unlikely to have significantly impacted the results.

Following recruitment and baseline data collection, participants underwent LFS and returned for clinical review and face-to-face data collection at 3, 6, and 12 months postsurgery. Continued eligibility was re-evaluated at 3 months against a priori criteria (e.g., infection, loosening of the metalware). Those participants with evidence of surgical complication were excluded. Missing data were treated as per protocol, a case wise deletion of the relevant variable and time point, which is acceptable when the data are missing at random. Recruitment, data collection, and all surgery occurred at a tertiary neurosurgical hospital.

\section{Outcome measures}

Background and anthropometric data were collected by selfreport and corroboration with hospital records as required.
Height in centimetres and weight in kilograms were measured at pre-assessment clinic with standard equipment.

\section{Primary outcomes (feasibility evaluation)}

Based on MRC guidance [11], the feasibility evaluation recorded; total LFS operations performed during the recruitment phase; number of eligible patients (with reasons for ineligibility); recruitment (with reasons for refusal); acceptability and characteristics of outcome measures; follow-up rates (with reasons for attrition); engagement with REFS (defined as attending $\geq 50 \%$ sessions, weekly home exercise records, and progressive exercise achievement).

\section{Secondary outcomes (clinical and economic evaluation)}

The clinical and economic impact of REFS were evaluated with selected quantitative measures: Oswestry Disability Index, version 2 (ODI) [16], a widely used self-reported measure of back related disability; pain self-efficacy questionnaire (PSEQ) [17], evaluating participants confidence in their ability to perform tasks despite pain; hospital 
Fig. 2 The REFS programme

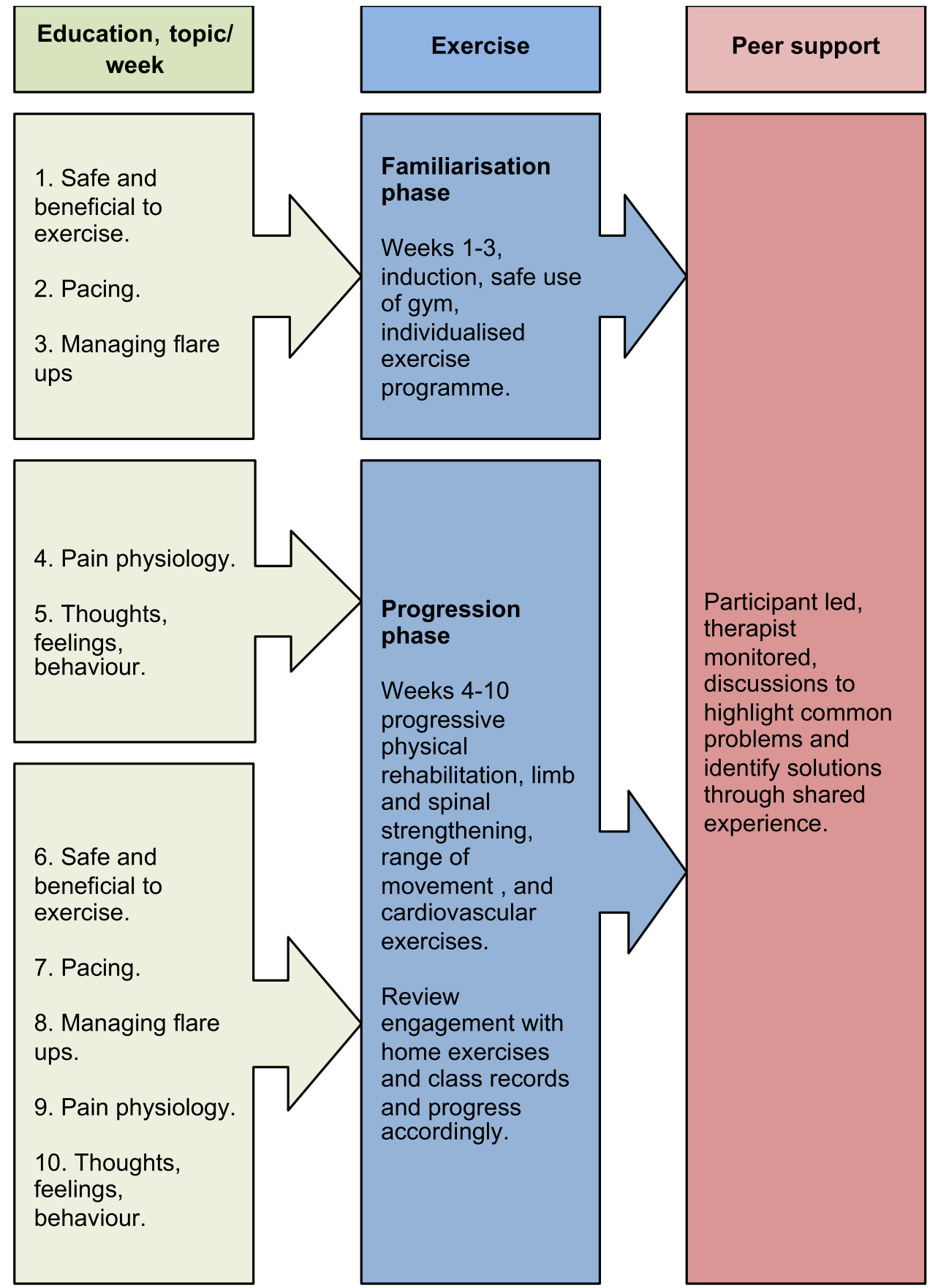

anxiety and depression scale (HADS) [18], a measure of hospital-related anxiety and depression; European Quality of Life 5 Dimensions Score (EQ-5D-5L) [19], a measure of health status across a range of indicators; aggregated functional performance time (AFPT) [20], the aggregated time in seconds for a participant to walk 50 yards, sit-tostand and walk 50 yards, and climb and descend a flight of 12 stairs. A 'nested' economic evaluation (REFS $n=10$, 'usual care' $n=10$ ) was conducted over the 12 -month study period employing the Client Services Receipt Inventory (CSRI) [21], a structured researcher-led interview collating relevant economic data, modified for use following LFS.

\section{Study intervention}

All participants received standard hospital-based postoperative care irrespective of group allocation (mobility assessment, anti-thrombotic exercises, check X-rays, and general medical/nursing care). Postoperative advice was provided to all participants, to be followed between discharge and the 3-month review; lift nothing heavier than a full kettle 
Fig. 3 Consort flow diagram for REFS study

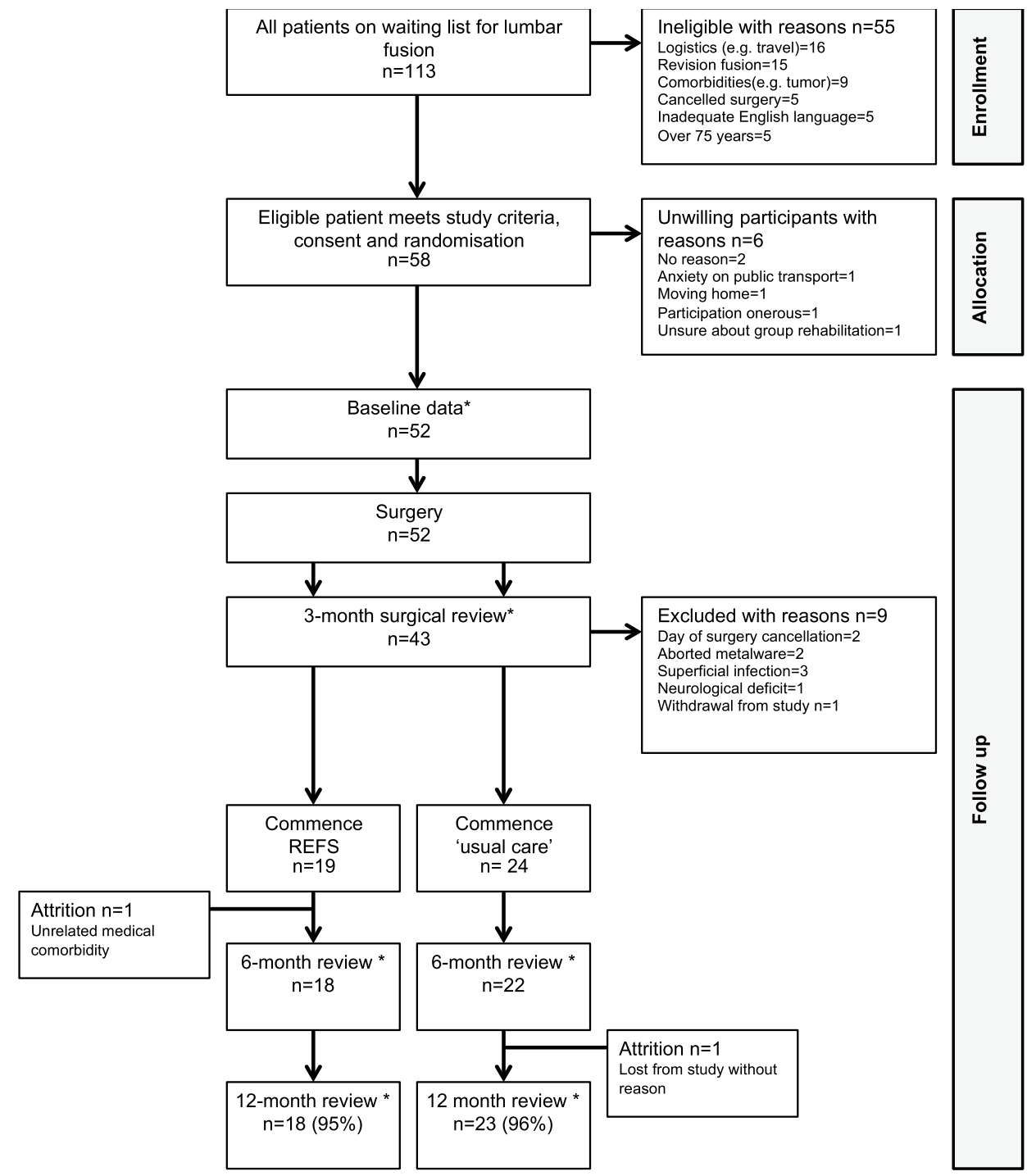

*=quantitative data collection. and progress outdoor walking ( $<2$ miles/day) commensurate with pain. Participants were discharged when the surgical team considered it safe to do so.

\section{REFS programme}

The REFS programme was delivered at a central London hospital by senior physiotherapists (band 7/8) who received 3 -h training in the delivery of REFS (intervention manual available from corresponding author). The programme consisted of 10 consecutive weekly, group $(n \leq 8)$ rehabilitation sessions ( $<90$ min duration). Considered deviations in the delivery of REFS were permitted to meet the specific requirements of individual participants, for example those with coexisting shoulder pathology were provided alternatives to the shoulder raise. Participants were provided with an exercise diary to set goals and record their activity/ adverse reactions to exercise, and an exercise sheet to facilitate home exercise.

Each session commenced with education ( $<20 \mathrm{~min})$, comprising of 5 predetermined topics, commencing with topic 1 (week 1) progressing to topic 5 (week 5). Each session commenced by briefly revisiting the topic(s) from previous week(s), thereby building the educational content throughout the programme. Following week 5, each topic was revisited to help participants apply the early learned principles into functional settings. Education was followed by supervised exercise (<60 min), comprising of 'low-tech' cardiovascular, range of movement, and limb and spine strengthening exercises. Conceptually, weeks 1-3 were considered the familiarisation phase and weeks 4-10 the progressive exercise phase. Each session concluded with a peer support discussion 
Table 1 Baseline participant characteristics on study entry

\begin{tabular}{|c|c|c|}
\hline Variable & REFS group $(n=25)$ & Usual care $(n=27)$ \\
\hline Gender $n=$ female $(\%)$ & $19 / 25(54.3 \%)$ & $\begin{array}{l}16 / 27 \\
(59.3 \%)\end{array}$ \\
\hline BMI & $28.26 \pm 7.39$ & $27.33 \pm 3.53$ \\
\hline Age & $55.9 \pm 13.5$ & $52.6 \pm 13.6$ \\
\hline $\begin{array}{l}\text { Symptom duration in } \\
\text { months }\end{array}$ & $100.8 \pm 87.0$ & $140.6 \pm 165.8$ \\
\hline $\begin{array}{l}\text { Previous spinal surgery } \\
\quad(y)\end{array}$ & $\begin{array}{l}10 \\
(40 \%)\end{array}$ & $\begin{array}{l}8 \\
(29.6 \%)\end{array}$ \\
\hline Smoker $(y)$ & $\begin{array}{l}3 \\
(12 \%)\end{array}$ & $\begin{array}{l}6 \\
(22.2 \%)\end{array}$ \\
\hline \multicolumn{3}{|l|}{ Work status } \\
\hline Employed (ft + pt) & $11(44 \%)$ & $11(41 \%)$ \\
\hline Home maker & $2(8 \%)$ & $1(4 \%)$ \\
\hline Benefits, ill health & $7(28 \%)$ & $10(37 \%)$ \\
\hline Retired & $5(20 \%)$ & $5(18 \%)$ \\
\hline \multicolumn{3}{|l|}{ Diagnosis } \\
\hline Primary disc disease & $3(12 \%)$ & $10(37 \%)$ \\
\hline Secondary disc disease & $6(24 \%)$ & $5(19 \%)$ \\
\hline Lytic spondylolisthesis & $3(12 \%)$ & $5(19 \%)$ \\
\hline $\begin{array}{l}\text { Degenerative canal } \\
\text { stenosis }\end{array}$ & $13(52 \%)$ & $7(26 \%)$ \\
\hline \multicolumn{3}{|l|}{ Number of levels fused } \\
\hline 1 & 17 & 21 \\
\hline 2 & 6 & 4 \\
\hline 3 & 1 & 2 \\
\hline Mean levels fused & $1.28 \pm 0.61$ & $1.29 \pm 0.6$ \\
\hline
\end{tabular}

Data are mean \pm SD or $n \pm(\%)$

Table 2 Descriptive baseline statistics by group

\begin{tabular}{lccl}
\hline Variable & $\begin{array}{l}\text { REFS mean } \pm \text { SD } \\
(n=25)\end{array}$ & $\begin{array}{l}\text { 'Usual care' } \\
\text { mean } \pm \text { SD } \\
(n=27)\end{array}$ & $p$ value $t$ test \\
\hline ODI & $49.6 \pm 15.42$ & $55.32 \pm 18.24$ & $p=0.238$ \\
EQ5D VAS & $50.42 \pm 18.16$ & $43.85 \pm 20.9$ & $p=0.243$ \\
EQ5D index & $0.5133 \pm 0.2715$ & $0.3557 \pm 0.3306$ & $p=0.071$ \\
HADS (a) & $10.64 \pm 4.33$ & $9.54 \pm 4.46$ & $p=0.375$ \\
HADS (d) & $8.72 \pm 4.94$ & $9.19 \pm 4.21$ & $p=0.715$ \\
PSEQ & $27.64 \pm 14.92$ & $24.23 \pm 14.37$ & $p=0.409$ \\
AFPT & $90.33 \pm 49.11$ & $107.32 \pm 60.74$ & $p=0.362$ \\
\hline
\end{tabular}

( $<15 \mathrm{~min}$ ) to highlight common problems and identify solutions through shared experience (Fig. 2).

\section{Usual care}

'Usual care' consisted of referral for local individual physiotherapy, commonly comprising 6 sessions (30 min) of face-to-face physiotherapy including individualised exercise-based self-management [10].

\section{Statistical analyses}

Statistical analyses were performed using Stata software (StataCorp LP. 2015. Stata Statistical Software: Release 14. College Station, TX). All tests were two-sided and assessed statistical significance at the $p<0.05$ level. Outcome distributions were checked to ensure parametric assumptions were met.

\section{Results}

Participants $(n=52)$ were recruited from 58 eligible patients. A consort flow diagram is provided reporting primary study aims including recruitment, retention, and data capture (Fig. 3). The reasons for exclusion (progressive neurological disorder $n=2$, severe osteoporosis, scoliosis, cerebral palsy, myelopathy $n=2$, intrinsic cord lesion, and malignancy) appeared related to the neurological specialism of the recruiting site. Background participant data are presented (Table 1). Of the 19 participants randomised to REFS, 18 (95\%) engaged with the programme (mean attendance 8.6 sessions). There was no crossover between groups.

Data capture was high (>95\%) for self-report outcomes, and aligning data collection with surgical follow-up may have enhanced this. Data capture for physical testing was inadequate at all follow-up time points for 'usual care' participants, for example at 12 months $34.8 \%$ of participants were unwilling/unable to undergo physical testing (AFPT) compared to $5.6 \%$ of REFS participants. This was primarily a consequence of ongoing pain/impaired mobility, those unwilling/unable to complete the AFPT had a higher mean (SD) disability (ODI) than those able to undertake physical testing $(57.42 \pm 15.33$ vs $28.17 \pm 21.45)$.

Secondary study aims relate to the clinical and economic impact of REFS. No significant difference existed between groups at baseline although there were more smokers (22.2\% vs $12 \%$ ) and participants with primary disc disease (37\% vs $12 \%$ ) in the 'usual care' group (Table 2). A minimal clinically important difference (MCID) of $10 \%$ has been reported for the ODI [22]. Therefore, REFS participants achieved a short-term unadjusted MCID $(-13.27 \% \pm 13.46)$, which was not observed in the 'usual care' group $(-2.42 \% \pm 12.33)$. This was maintained in the longer term $(-14.72 \% \pm 13.34$ vs $-7.57 \% \pm 13.91)($ Table 3$)$.

Between-group evaluation was conducted with multilevel regression analyses, adjusted for smoking status $(p=0.001)$, body mass index $(p=0.883)$, and baseline 
Table 3 Unadjusted mean \pm SD change from baseline -3 month, 3-6 months, and 3-12 months with adjusted between-group $p$ values

\begin{tabular}{|c|c|c|c|c|c|c|c|c|c|}
\hline & \multicolumn{2}{|c|}{$\begin{array}{l}\text { Unadjusted mean change base- } \\
\text { line- } 3 \text { months }\end{array}$} & \multirow{2}{*}{$\begin{array}{l}\text { Between- } \\
\text { group } \\
\text { adjusted } p \\
\text { values }^{\text {a }}\end{array}$} & \multicolumn{2}{|c|}{ Unadjusted mean change $3-6$ months } & \multirow{2}{*}{$\begin{array}{l}\text { Between- } \\
\text { group } \\
\text { adjusted } p \\
\text { values }^{\mathrm{a}}\end{array}$} & \multicolumn{2}{|c|}{ Unadjusted mean change $3-12$ months } & \multirow{2}{*}{$\begin{array}{l}\text { Between- } \\
\text { group } \\
\text { adjusted } p \\
\text { values }^{\mathrm{a}}\end{array}$} \\
\hline & REFS & Usual care & & REFS & Usual care & & REFS & Usual care & \\
\hline ODI & $-4.0 \pm 16.59$ & $-11.42 \pm 19.99$ & $p=0.19$ & $-13.27 \pm 13.46$ & $-2.42 \pm 12.33$ & $p=0.014 *$ & $-14.72 \pm 13.34$ & $-7.57 \pm 13.91$ & $p=0.101$ \\
\hline EQ5D VAS & $12.72 \pm 21.59$ & $19.55 \pm 22.0$ & $p=0.45$ & $6.16 \pm 17.51$ & $-2.68 \pm 16.47$ & $p=0.062$ & $6.72 \pm 14.84$ & $-1.76 \pm 18.01$ & $p=0.106$ \\
\hline EQ5D index & $0.1274 \pm 0.2818$ & $0.2082 \pm 0.2796$ & $p=0.29$ & $0.0628 \pm 0.1310$ & $-0.0177 \pm 0.131$ & $p=0.121$ & $0.0214 \pm 0.1683$ & $-0.0211 \pm 0.1799$ & $p=0.402$ \\
\hline HADS (a) & $-1.84 \pm 3.56$ & $-1.9 \pm 2.91$ & $p=0.968$ & $0.61 \pm 4.19$ & $0.05 \pm 3.15$ & $p=0.386$ & $-0.38 \pm 4.34$ & $0.42 \pm 3.18$ & $p=0.432$ \\
\hline HADS (d) & $-2.68 \pm 4.89$ & $-1.76 \pm 4.1$ & $p=0.41$ & $-0.55 \pm 4.55$ & $1.21 \pm 2.95$ & $p=0.394$ & $-0.38 \pm 5.11$ & $-0.04 \pm 2.37$ & $p=0.952$ \\
\hline PSEQ & $3.26 \pm 18.28$ & $9.42 \pm 12.86$ & $p=0.177$ & $8.35 \pm 10.78$ & $-0.55 \pm 13.33$ & $p=0.007 *$ & $4.66 \pm 12.08$ & $2.66 \pm 9.35$ & $p=0.49$ \\
\hline AFPT & $-23.7 \pm 53.96$ & $4.25 \pm 15.88$ & $p=0.1$ & $-19.37 \pm 29.05$ & $-7.75 \pm 25.41$ & $p=0.62$ & $-17.90 \pm 37.29$ & $-27.22 \pm 33.03$ & $p=0.492$ \\
\hline
\end{tabular}

${ }^{a}$ Multilevel regression for depression, smoking status, and body mass index

*Statistically significant $p<0.05$

depression $(p<0.001) R^{2}=59.7 \%$ for all outcome measures from baseline (preoperative) - 3 months, 3-6 months (short-term post-rehabilitation), and 3-12 months (longterm post-rehabilitation) (Table 3). Participants allocated to REFS achieved statistically significant improvements in short-term adjusted mean disability $(p=0.014)$ and pain self-efficacy $(p=0.007)$. Short (6-month) and longer-term (12-month) effect sizes $(95 \% \mathrm{CI})$ for disability (ODI) for were calculated for REFS $0.63(-0.03$ to -1.28$)$ and 0.69 $(0.24$ to -1.35$)$ and 'usual care' $-0.02(-0.63$ to -0.57$)$ and $0.15(-0.43$ to -0.75$)$. Therefore, REFS achieved a medium effect $(d>0.50)$ in both short- and longer-term disability, which was not evident in the 'usual care' group.

Evidence was also observed of nonsignificant, but potentially important, improvements in quality of life and physical function at all postoperative time points favouring REFS over 'usual care' (Fig. 4).

Economic evaluation employed linear regression analyses, by group and baseline costs, with bootstrapping to account for the skewed distribution of cost data, for calculation of QALY's. Total QALYs in the REFS group were higher than the 'usual care' group after adjustment for baseline differences in EQ5D-5L scores, revealing an adjusted mean (SD) difference of $0.03( \pm 0.064), p=0.637$. Therefore, REFS provided 0.03 more QALYs at an additional cost of $£ 92$ per patient. The indicative cost per QALY was $£ 3067$, below the NICE threshold of $£ 20-£ 30,000$. Disaggregated unadjusted mean costs $(\mathfrak{E})$ by group are provided (supplemental material).

\section{Discussion}

This is the first study to compare the feasibility of delivering a theoretically informed rehabilitation programme, REFS, with 'usual care' (individualised physiotherapy) following technically successful LFS. Primary outcomes reporting the feasibility of REFS were encouraging, including recruitment $(n=52 / 58)$ and retention $(>95 \%)$ of eligible participants, posing little threat to internal validity. Engagement has been described as a co-constructed process of participation, contribution, retention, and attendance [23]. Applying these criteria, 95\% of participants engaged with REFS.

Ineligibility appeared related to the neurological specialism of the hospital, and future studies should include orthopaedic and neurosurgical spine units.

Feasibility studies should not focus on extensive betweengroup analyses in unpowered samples. However, the secondary study aims, related to clinical impact, suggest REFS achieved clinically meaningful improvements in short- and longer-term disability, which reached statistical significance in the short term $(p=0.014)$. The short- and longer-term effect sizes for REFS participants ( 0.63 and 0.69 , respectively) were comparable to those of Abbott et al. [7] (0.56 and 0.58) but smaller than those reported by Monticone et al. [8] (1.13 and 1.08). This is potentially justified as the report by Monticone et al. included the combined surgical and rehabilitation effects.

The observed improvements demonstrated in the current study are likely to be attributable to the content and delivery of REFS, which was designed to reduce disability through enhanced self-efficacy. This substantiates the social cognitive theory [15] as the overarching programme theory, in which self-efficacy is afforded primacy. However, by 12 months the pain self-efficacy scores between groups had converged. It is plausible that enhanced self-efficacy may be a group-mediated effect, which Bandura described as the collective efficacy of group interventions [24]. A recent similar study in which rehabilitation was delivered individually as opposed to a group setting demonstrated no significant between-group differences [25]. This finding potentially reinforces the critical relevance of the group effect. A process evaluation, including a nested qualitative evaluation of participants experience of REFS, is currently underway to 

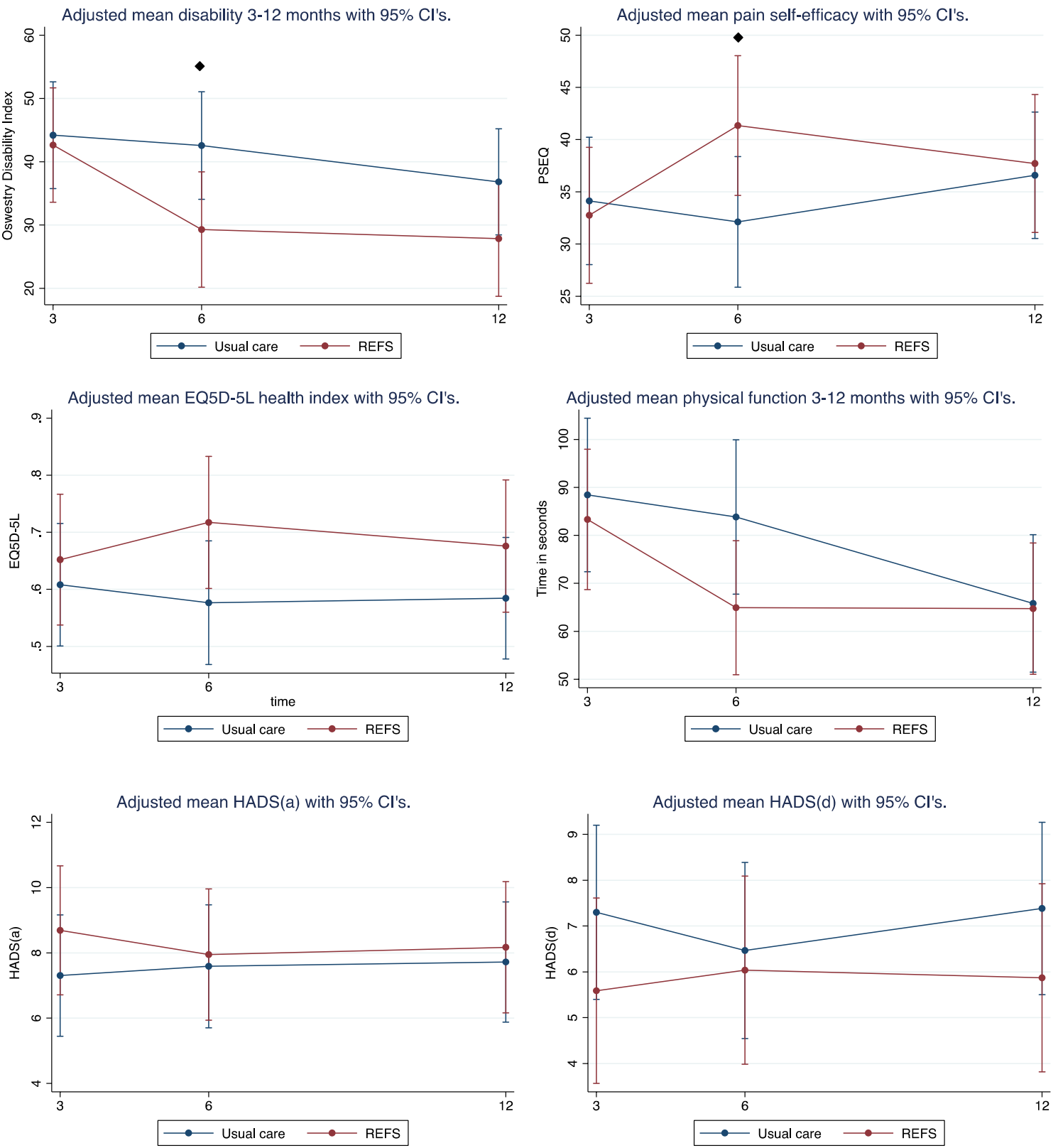

$\checkmark=$ Statistically significant at $p<0.05$.

Fig. 4 Adjusted mean with 95\% CI's for each variable by group

highlight valued content and explore the divergent numerical outcomes.

The REFS programme can be delivered in uni-professional settings by experienced physiotherapists with minimal extra training and appears affordable, with sensitivity analysis where half the participants are not allocated to another treatment arm, suggesting a total cost of $£ 137.50$ per participant.

\section{Study limitations}

The study findings were limited by sample size, the singlecentre design, and the reporting of 'usual care.' Readers should consider these aspects when interpreting the results. Data capture for physical testing (AFPT) was incomplete, introducing selection bias. A new mechanism for the evaluation of physical performance is required; the 6-min walk test and sit-to-stand 30 are proposed as performance rather than 
time becomes the dependant variable, potentially increasing acceptability.

The current study had 9 dropouts at 3 months, primarily due to technical surgical concerns, e.g., wound infection. Whilst it may appear preferable to defer recruitment until a satisfactory 3-month surgical review, this would result in a loss of critical preoperative data. Therefore, this surgical attrition should be included in future sample size calculations.

\section{Conclusion}

In conclusion, this study suggests that REFS provides a feasible and affordable alternative to current 'usual care.' The REFS programme appears to convey a meaningful clinical improvement in functional ability. This improvement may be mediated by a group effect, which enhanced short-term self-efficacy. To adequately power a future efficacy study (assuming a two-sided significance of 5\%, 90\% power, a mean clinically important difference in disability between groups of $10 \%$, and a standard deviation of \pm 17.02 , derived from this study), two groups of $n=62$ participants would be required. Allowing for a $20 \%$ rate of attrition, this would equate to a sample of $n=155$. Such a study should include participants undergoing primary and revision fusion surgery, have no upper age limit, and include a more acceptable evaluation of physical performance.

Acknowledgements This article/paper/report presents independent research funded by the National Institute for Health. Research (NIHR). The views expressed are those of the author(s) and not necessarily those of the NHS, the NIHR or the Department of Health

Source of support Clinical Doctoral Research Fellowship awarded to the corresponding author from the National Institute of Health Research (NIHR).

\section{Compliance with ethical standards}

Conflict of interest All authors declare that they have no conflict of interest.

OpenAccess This article is distributed under the terms of the Creative Commons Attribution 4.0 International License (http://creativeco mmons.org/licenses/by/4.0/), which permits unrestricted use, distribution, and reproduction in any medium, provided you give appropriate credit to the original author(s) and the source, provide a link to the Creative Commons license, and indicate if changes were made.

\section{References}

1. Gibson JN, Waddell G (2005) Surgery for degenerative lumbar spondylosis: updated Cochrane Review. Spine 30(20):2312-2320
2. Deyo RA et al (2005) United States Trends in lumbar fusion surgery for degenerative conditions. Spine 30:1441-1445

3. United Nations (2015) World population ageing. Department of Economic and Social Affairs, Population Division. http://www. un.org/en/development/desa/population/publications/pdf/ageing/ WPA2015_Report.pdf. Accessed 18 Feb 2019

4. Stromqvist B, Fritzell P, Hagg O, Jonsson B (2007) Follow-up of lumbar surgery in Sweden 2007, The Swedish National Spine Register. The Swedish Spinal Surgery Society. Eur Spine J 22(4):953-974. https://doi.org/10.1007/s00586-013-2758-9

5. Abbott AD, Hedlund R, Tyni-Lenne R (2011) Patients' experience post-lumbar fusion regarding back problems, recovery and expectations in terms of the International Classification of Functioning, Disability and Health. Disabil Rehabil 33(15-16):1399-1408

6. Greenwood J, McGregor A, Jones F, Mullane J, Hurley M (2016) Rehabilitation following lumbar fusion surgery: a systematic review and meta-analysis. Spine 41(1):E28-E36

7. Abbott AD, Tyni-Lenne R, Hedlund R (2010) Early rehabilitation targeting cognition, behavior, and motor function after lumbar fusion: a randomized controlled trial. Spine 35(8):848-857

8. Monticone $\mathrm{M}$ et al (2014) Management of catastrophising and kinesiophobia improves rehabilitation after fusion for lumbar spondylolisthesis and stenosis. A randomised controlled trial. Eur Spine J 23(1):87-95

9. Oestergaard LG et al (2012) The effect of early initiation of rehabilitation after lumbar spinal fusion: a randomized clinical study. Spine 37(21):1803-1810

10. Rushton A, Heneghan N, Heap A, White L, Eveleigh G, Wright C (2014) Survey of current physiotherapy practice for patients undergoing lumbar spinal fusion surgery in the UK. Spine 39(23):E1380-E1387

11. Craig P, Dieppe P, Macintyre S, Michie S, Nazareth I, Petticrew $M$ (2008) Developing and evaluating complex interventions: the new Medical Research Council guidance. BMJ 337:1655-1660

12. Borek AJ et al (2015) A checklist to improve reporting of groupbased behaviour-change interventions. BMC Public Health 15(1):963

13. Boutron I et al (2008) Extending the CONSORT statement to randomized trials of non pharmacologic treatment: explanation and elaboration. Ann Intern Med 148(4):295-309

14. Michie S, van Stralen M, West R (2011) The behaviour change wheel: a new method for characterising and designing behaviour change interventions. Implement Sci 6:42

15. Bandura A (1986) Social foundations of thought and action: a social cognitive theory. Prentice-Hall Inc, Englewood Cliffs

16. Fairbank J, Pynsent $P$ (2000) The Oswestry disability index. Spine 25:2940-2953

17. Nicholas MK (2007) The pain self efficacy questionnaire: taking pain into account. Eur J Pain 11:153-163

18. Zigmund A, Snaith R (1983) The hospital anxiety and depression scale. Acta Psychiatr Scandanavica 67:361-367

19. Group, E. (1990) EuroQol-a new facility for the measurement of health-related quality of life. Health Policy 16:199-208

20. Hurley MV et al (1997) Sensorimotor changes and functional performance in patients with knee oseoarthritis. Arthritis Care Res 56:641-648

21. Beecham J, Knapp M (2001) Costing psychiatric interventions. Gaskell, London

22. Hagg O, Fritzell P, Nordwall A (2003) The clinical importance of changes in outcome scores after treatment for chronic low back pain. Eur Spine J 12:12-20

23. Bright FA et al (2015) A conceptual review of engagement in healthcare and rehabilitation. Disabil Rehabil 37(8):643-654

24. Bandura A (2000) Exercise of human agency through collective efficacy. Curr Dir Psychol Sci 9:75-78 
25. Ilves O et al (2017) Quality of life and disability: can they be improved by active postoperative rehabilitation after spinal fusion surgery in patients with spondylolisthesis? A randomised controlled trial with 12-month follow-up. Eur Spine J 26(3):777-784
Publisher's Note Springer Nature remains neutral with regard to jurisdictional claims in published maps and institutional affiliations.

\section{Affiliations}

\section{James Greenwood ${ }^{1}\left[\right.$ - Alison McGregor ${ }^{2} \cdot$ Fiona Jones $^{3} \cdot$ Michael Hurley $^{3}$}

$\triangle$ James Greenwood

james.greenwood4@nhs.net

1 Victor Horsley Department of Neurosurgery, National Hospital for Neurology and Neurosurgery, Internal Box 8, Queen Square, London WC1 3BG, UK
2 Biodynamics Lab, Charing Cross Hospital, Imperial College London, Charing Cross Campus, London, UK

3 Faculty of Health and Social Care Sciences, St Georges University of London, London, UK 